\title{
BER PERFORMANCE ANALYSIS OF 4QAM/OFDM OVER DIFFERENT SCENARIOS OF LAPLACE FADING CHANNEL.
}

\author{
*Hassan F. Mohammed ${ }^{1}$
}

\author{
Ghanim A. Al-Rubaye ${ }^{1}$
}

1) Electrical Engineering Department, College of Engineering, Mustansiriyah University, Baghdad, Iraq

\begin{abstract}
Multicarrier transmission, also known as (OFDM) Orthogonal Frequency Division multiplexing, in wireless communications, it has been proven to be an essential technique for countering multipath fading. It has been used successfully for HF radio applications and has been selected as the interface for digital audio transmission, digital terrestrial TV broadcasting, and highspeed wireless local area networks in Europe. In this paper, we suggested a new design for modeling multipath fading channels, such as the Laplace fading channel, in order to discover new simulation results and effects. Furthermore, the variance of the Laplace fading channel has been computed and the new Bit Error Rate (BER) derivation is established, and the performance of ( $M$ QAM), M-ary Quadrature Amplitude Modulation (with $\mathrm{M}=4$ over OFDM system under Laplace fading channels in Additive White Gaussian Noise (AWGN) is discussed and compared to the conventional M-QAM/OFDM system Rayleigh fading channel in AWGN. All the simulation results are examined using the optimum signal detection based on the Euclidean distance and evaluated using Monte-Carlo simulation.
\end{abstract}

Keywords: OFDM, Laplace Fading Channels, Rayleigh Fading Channels, BER, Euclidean Distance

\section{Introduction}

In wireless communications, the efficiency of the transmission and receiving systems is critical. Digital modulation schemes have the benefit of increasing wireless network power, efficiency, and consistency. OFDM is essential in systems of wireless and mobile communication because OFDM systems can deal with the impact of channel environment[1].

The basic concept behind OFDM is to divide a high-rate data channel for a number of lower-rate sources that are concurrently transmitted by a numbers of subcarriers. The proportional amount of time dispersion produced by multipath delay spread decreases as the symbol duration for lower rate parallel subcarriers rises. Intersymbol interference (ISI) is virtually eliminated by using a guard time in every OFDM signal. The OFDM symbol is cyclically extended during the guard time to prevent Intercarrier Interference (ICI)[2]. System's usable bandwidth, OFDM has been adopted in several recent wireless applications. It has been implemented for European Digital Audio Broadcasting (DAB) and Digital Video Terrestrial Broadcasting (DVB) standards, proposed for UMTS (Universal Mobile Telecommunication Systems), and recently standardized for modern cellular LAN generations (HIPERLAN: High Performance Radio LAN) [3]. Several previous research papers have analyzed and discussed the performance of BER of OFDM with various

*Corresponding Author: hassn0eng@gmail.com 
digital modulation schemes under various fading channel scenarios. In[4], On mobile channels, an OFDM system was implemented, and its output was investigated in the flat fading situation. In [3], analyzes the performance of OFDM structures in the presence and absence of a phase correction function, as well as its reliance on the number of subcarriers.in[5] To combat the difficult environment of power-line communication (PLC) channels, they suggested Irregular Non-Binary Low-Density Parity Check (IR-NB-LDPC) codes with a Signed Log Fast Fourier Transform (SL-FFT) decoding method. The Sum Product algorithm (SPA)is used to compare their performance to that of Irregular Binary LDPC (IR-B-LDPC) codes.in [6], When impulsive noise is present, the OFDM performance for physical layer network coding system has been improved based on turbo codes over multipath power line communication channels. In[7], the performance of irregular low-density parity search (LDPC) coded OFDM using 4096 QAM is examined. and improved based on optimal detectors. $\operatorname{In}[1]$, the performance of BER to OFDM and M-QAM modulation schemes has been examined in AWGN and Rayleigh channels. in [8] The efficient complex-valued ratio distributions of the individual and combined samples of noise at the zero-forcing ( $\mathrm{ZF}$ ) equalizer output were calculated based on the obtained efficient complex-valued ratio distributions. The studies of performance of the proposed turbo-coded orthogonal frequency division multiplexing (TCOFDM) are examined over the frequencyselective power-line communication with lognormal channel gain.in[9], The BER tests of M-ary offset QAM for bank of filter based multicarrier (M-ary OQAM/FBMC)) was investigated over various fading channel scenarios, including unshadowed LOS Rician K-factor (RK-f) channel of fading, nonline-ofsight (NLOS) shadowed fading model, and compound model of unshadowed/shadowed fading.

This paper evaluates the BER performance of 4QAM/OFDM over different scenarios of Laplace fading channel in AWGN by using an optimal detector based on the closest signal to the received signal (Euclidean distance).

The major contributions of this paper are related to BER Performance analysis of 4QAM/OFDM over different scenarios of Laplace fading channel are as follows:

The effective probability distribution and the effective SNR distribution of the Laplace fading channel in the frequency domain at the OFDM demodulator output are derived with the help of the central limit theorem (CLT).

We derive the BER based on the effective SNR distribution of the Laplace fading channel in the frequency domain.

We detect the received signal by using an optimum detector based on the Euclidean distance depending on the derived effective probability distribution at the OFDM demodulator output.

The rest of this paper is organized as follows: Section 2 discuss the multipath fading channel ,the model of the system presented in Section 3,Section 4 the derivation of the BER performance over 4QAM/OFDM system under Laplace fading channel, Section 5 discuss the simulated results, Section 6 for conclusion finally Section 7 for references.

\section{Multipath Fading Channel}

Multiple transmission paths from a transmitter to a receiver are common in an atypical wireless communication system due to scattering by various Obstacles. Signal copies traveling down various paths can experience varying degrees of attenuation, distortion, delay, and phase change. As a result, at the receiver, Interference, both constructive and destructive, will occur. When 
there is destructive interference, the signal strength is greatly reduced. This is referred to as fading [10]. The expression of "fading" refers to the rapid variations in the intensity of a received signal of radio within a brief period of time[11]. Fading will severely degrade a system's efficiency (in terms of error probability). Not only do multiple propagation paths occur frequently, particularly in mobile communications, but they are also time-varying. As a consequence, a time-varying fading channel is established [10]. The overall received signal is a vector number of independently delayed signals, the relative phase angles of which depend on the frequency as well as the amplitudes and delays of echo. Due to the fact that the amplitudes and delays of echos vary with time, significant differences in obtained signal intensity at a particular frequency as a function of time or strength at a specified time as a function of frequency are observed; the latter is referred to as "selective fading". Another consequence of multipath reception is the received signal's delay spread, including numerous components that arrive at separate times with a difference in arrival time greater than period of one symbol (Ts), the signal components of the symbol present at the receiver input may be hampered by components of previously transmitted symbols. This is known as Intersymbol Interference (ISI). Furthermore, channel time difference is caused by Doppler spread and is understood by fast or slow fade; in the frequency domain, signal interference caused by fast fading increases as Doppler spread increases, allowing the channel impulse response to shift rapidly throughout the symbol length. Because the Doppler spread is substantially less than the baseband bandwidth, it mostly results in a loss of SNR[12], [13]. As a basis for multipath fading channels, previous work indicates a Rayleigh fading channel and a Rician fading channel. We suggested a new model (Laplace fading channel) in this paper to find new simulations and effects.

\section{System Model}

In this paper, M-QAM modulation is applied to the generated signal. The serial modulated complex data is then transformed to $\mathrm{n}$ parallel data streams, each of which contains discrete frequency signals. The Inverse Fast Fourier Transform (IFFT) transforms these signals into time samples in the message length sequence (N). Equation (1) denotes the sampling function in multicarrier system signals.

$x[n]=\frac{1}{\sqrt{N}} \sum_{i=0}^{N-1} X[i] e^{\frac{j 2 \pi n i}{N}}$

The cyclic prefix (CP) code is produced and inserted into OFDM symbols before being translated to data streams in serial form, which is $\mathrm{x}$ [n] as stated in the equation (1). This signal is combined by additive noise in the channel of wireless, yielding the signal seen in equation (2)

$y=h * x+w$

Where the complex random variable $h$ represent the discrete impulse response coefficients in the time domain of a multipath fading channel and $w$ is the AWGN. It was already stated that the multipath fading channel for wireless communication was modeled Laplace distribution [14] in Laplace fading channel, when the random variable for fading magnitude $h$ fits a Laplace distribution in the time domain, the PDF will be distributed as seen in [14] as

$f(h)=\frac{1}{2 b} e^{-\frac{|h-a|}{b}},-\infty<\mathrm{h}<\infty$

Where, $a \in(-\infty, \infty), b>0$ are location and scale parameters respectively with mean and variance equal to a and $2 b^{2}$, respectively as in [15]. The Laplace distribution over different scenarios of location parameter and scale parameters can be shown in Fig. 1. 


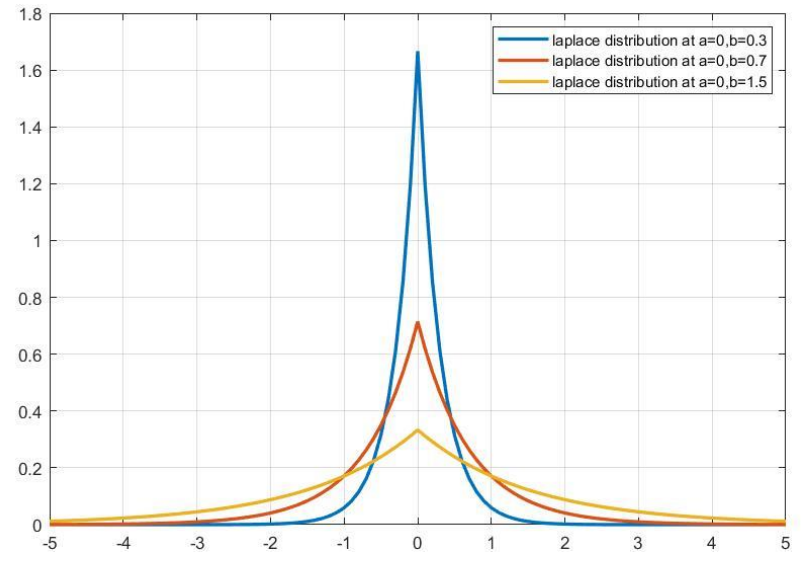

Figure 1. The Laplace distribution

The modulated signal is processed using the 64-point IFFT technique. Following that, $16 \mathrm{CP}$ symbols are inserted and relayed through a channel. The CP length is more than the channel's maximum delay spread. The received signal is combined with the AWGN signal and processed using $\mathrm{CP}$ elimination and the Fast Fourier Transform (FFT).

The Maximum Likelihood (ML) detection can be implemented by finding the minimum Euclidean distance between all the possible transmitted symbols over the Laplace fading channel $\mathrm{H}$ in the frequency domain and the received signal $\mathrm{Y}$ as [16]:

$\bar{d}=\arg \min _{X \in C}\|Y-H . X\|^{2}$

Where $\mathrm{Y}$ is the complex received noisy signal in the frequency domain and $C=\left\{C_{1}, C_{2}, C_{3}, C_{4}\right\}$ represents the collection of all possible modulated symbols $\mathrm{X}$ in a 4QAM constellation as shown in Fig. 2.

Finally, The BER is calculated by XORing the signal of information at the transmitter with the finally ultimately signal, i.e. (sum of error bits in $\bar{d}$, and then dividing the value by the amount of transmitted bits $d[10]$.

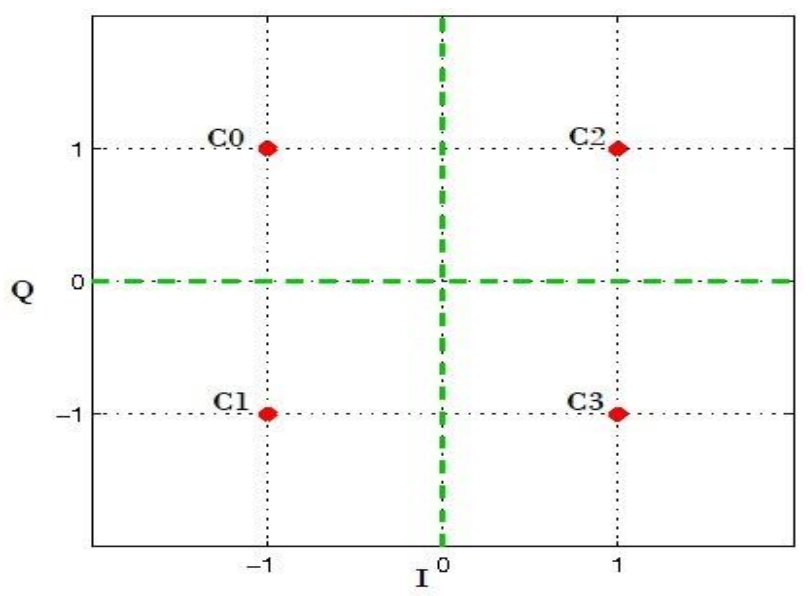

Figure 2. 4QAM constellation

Fig. 3 depicts the setup of an OFDM transceiver system (transmitter and receiver).

OFDM Tx.

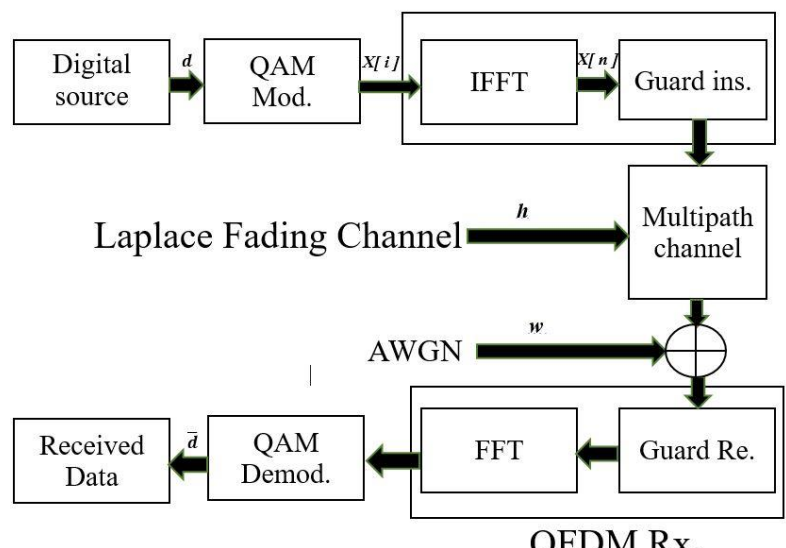

OFDM Rx.

Figure 3. Frame diagram for an OFDM radio transceiver system.

\section{The BER Derivation Performance Over 4QAM/OFDM System Under Laplace Fading Channel}

The fading effect is calculated using various modulation schemes. The probability of error in bit $(\mathrm{Pb})$, also known as the BER, is a more precise efficiency metric for calculating modulation schemes. The following integral can be used to calculate the BER output of any digital modulation scheme in a slow flat fading path [17]

$P_{b}=\int_{0}^{\infty} P_{b, A W G N}(\gamma) P_{d f}(\gamma) d \gamma$ 
When the signal-to-noise ratio is set to a certain value, the error probability of a certain modulation scheme in an AWGN channel is $P_{b, A W G N}(\gamma)$ where

$\gamma=h^{2} \frac{E_{b}}{N_{o}}$

In a non-fading of AWGN channel, $h$ is the random variable of channel gain, $\frac{E_{b}}{N_{o}}$ is the bit energy to noise power density ratio, the fading channel's instantaneous power is denoted by $\mathrm{h}^{2}$ and $P_{d f}(\gamma)$ is the fading channel's probability density function of $\gamma$.

\subsection{BER of 4QAM Modulation in AWGN Channel}

The BER for M-PSK in a channel of additive white Gaussian noise (AWGN) is provided by [17]

$$
B E R_{M-P S K}=\frac{2}{\log _{2} M} Q\left(\sqrt{\frac{2 E_{b} \log _{2} M}{N_{0}}} \sin \frac{\pi}{M}\right)
$$

4-PSK is also known as quadrature phase shift keying (QPSK) and is equivalent to MQAM with $\mathrm{M}=4[18]$.

So for MQAM coherent identification, equation (7) with $\mathrm{M}=4$ reduces to:

$$
B E R_{M Q A M}=Q\left(\sqrt{\frac{2 E_{b}}{N_{0}}}\right)
$$

Equation (8) may be rewritten as follows:

$$
B E R_{4 Q A M, A W G N}=\frac{1}{2} \operatorname{erfc}\left(\sqrt{\frac{E_{b}}{N_{0}}}\right)
$$

\subsection{Novel Derivation of BER Of 4QAM Modulation in Laplace Fading Channel}

The distribution of the signal to noise ratio in the frequency domain can be expressed based on the Classical Central Limit theorem (CLT) as

$P_{d f}(\gamma)=\frac{1}{\bar{\gamma}} \exp \left(-\frac{\gamma}{\bar{\gamma}}\right)$
Where $\bar{\gamma}=\frac{E_{b}}{N_{o}} E\left[h^{2}\right]$ represent the signal-tonoise ratio on average. For $E\left[h^{2}\right]=1, \bar{\gamma}$ represents the fading channel's average $\frac{E_{b}}{N_{o}}$.

Classical (CLT) It asserts that if $\mathrm{G}$ is equal to

$$
\frac{1}{n} \sum_{i=1}^{n} V_{i}
$$

and $\left\{V_{i}, i=1,2, \ldots, n\right\}$ is a series of independent and identically distributed (iid) random variables with $m=E\left[V_{1}\right]<\infty$ and $\sigma^{2}=\operatorname{VAR}\left[V_{1}\right]<\infty$, then the PDF of $\mathrm{G}$ approaches the Gaussian distribution as $n \rightarrow \infty$ regardless of the pdf of $\mathrm{V}$ with mean $\mathrm{m}_{\mathrm{G}}=\mathrm{m}_{\mathrm{v}}$ and variance $\sigma_{G}^{2}=\sigma_{V}^{2} / n$ [19].

Let $2 b^{2}=L$ and $\frac{E_{b}}{N_{o}}=R$ so equation (10) can written as:

$P_{d f}(\gamma)=\frac{1}{L R} e^{\frac{-\gamma}{L R}}$

The BER of AWGN in the presence of channel $h$ is

$P_{b, A W G N}(\gamma)=\frac{1}{2} \operatorname{erfc}(\sqrt{\gamma})$

Sub equation (13) and equation (12) in equation (5) we get:

$$
P_{b}=\int_{0}^{\infty} \frac{1}{2} \operatorname{erfc}(\sqrt{\gamma}) * \frac{1}{L R} e^{\frac{-\gamma}{L R}} d \gamma
$$

$$
\begin{gathered}
=\left(-\frac{0.5 \sqrt{L R} \operatorname{erf}\left(\frac{\sqrt{\gamma(L R+1)}}{\sqrt{L R}}\right)}{\sqrt{L R+1}}\right. \\
\left.-\frac{1}{2} \operatorname{erfc}(\sqrt{\gamma}) e^{\frac{-\gamma}{L R}}\right) \infty \\
=\left(-\frac{0.5 \sqrt{L R} \operatorname{erf}(\sqrt{\infty})}{\sqrt{L R+1}}-\frac{1}{2} \operatorname{erfc}(\sqrt{\infty}) e^{-\infty}\right)- \\
\\
\left(-\frac{0.5 \sqrt{L R} \operatorname{erf}(\sqrt{0})}{\sqrt{L R+1}}-\frac{1}{2} \operatorname{erfc}(\sqrt{0}) e^{0}\right)
\end{gathered}
$$




$$
\begin{aligned}
& =-\frac{0.5 \sqrt{L R}}{\sqrt{L R+1}}-(-0.5) \\
& =0.5\left[1-\sqrt{\frac{L R}{L r+1}}\right]
\end{aligned}
$$

So

$$
\mathrm{Pb}=0.5\left[1-\sqrt{\frac{\left(2 b^{2}\right) \frac{E_{b}}{N_{o}}}{\left(2 b^{2}\right) \frac{E_{b}}{N_{o}}+1}}\right]
$$

\section{Simulation Results}

The BER performances of 4QAM/OFDM over Laplace fading channels in AWGN was examined using a MATLAB simulation software. The following simulation parameters were set, the number of sub-carriers $\mathrm{N}=64$ and $\mathrm{CP}=16$, the size of constellation was set as 4QAM.

\subsection{Performance of 4-QAM/OFDM System Over Laplace Fading Channel}

The performance of BER of 4QAM modulation in the OFDM system over Laplace fading channel in AWGN is shown in Fig. 4 Which is compared with The performance of BER of 4QAM modulation in the OFDM system over Rayleigh fading channel in AWGN. In this simulation, We were able to transmit 52 subcarriers utilizing an OFDM method based on a 64-point IFFT circuit with a guard time period equal to $1 / 4$ time of symbol after inserting 6 zeros at the beginning of data, one zero in the middle, and five zeros at the end to present over sampling. The following figures show the effect of adjusting the (a) and (b) parameters on the BER performance over Laplace fading channels compared to Rayleigh fading channels.

Simulations were run with various values of the channel effect by changing the scale parameter $b$ $=0.3,0.7,1.5$ for severe impact, normal impact and low impact, respectively, and for the location parameter $\mathrm{a}=0$, as follows:

1. Based on the first value $(b=0.3)$, the performance of BER of the system over a Laplace fading channel is worse than that of the system over a Rayleigh fading channel over all values of $\frac{E_{b}}{N_{o}}$ in $\mathrm{dB}$ as shown in Fig.4

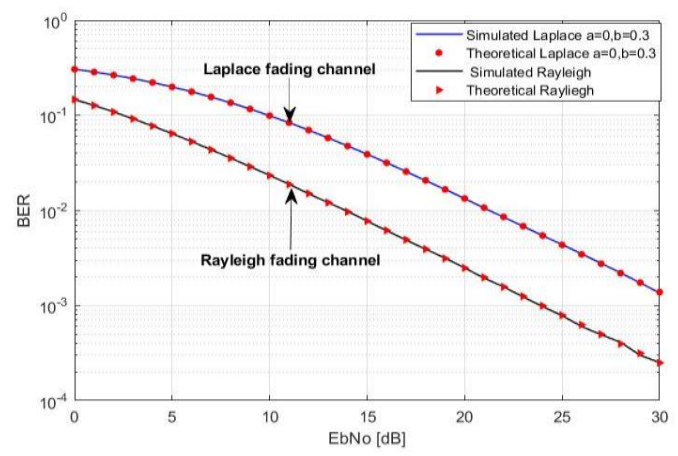

Figure 4. The performance BER of Laplace fading channel with $b=0.3$

2. Furthermore, at the second value $(b=0.7)$, the performance of BER of the system over a Laplace fading channel is comparable to or similar to that the system over a Rayleigh fading channel as shown Fig.5.

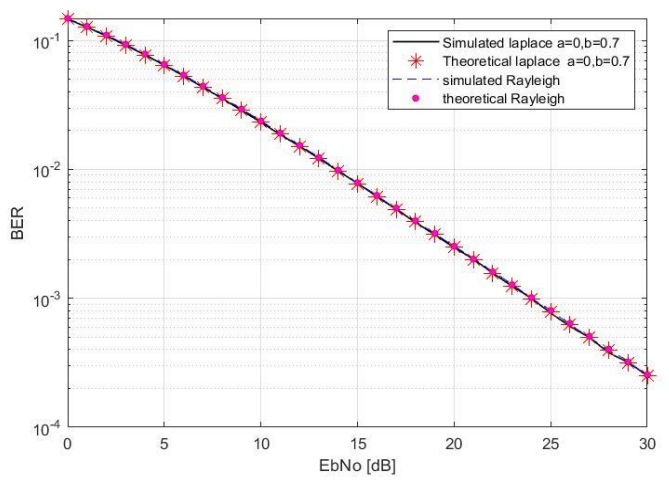

Figure 5. BER performance of Laplace fading channel with $b=0.7$

3. Finally, the third value $(b=1.5)$, the performance of BER of the system over a Laplace fading channel is better than that of the system over a Rayleigh fading channel as shown in Fig.6. 


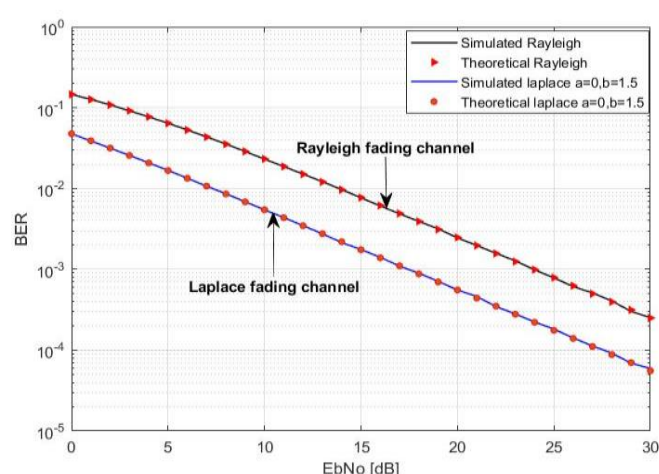

Figure 6. BER performance of Laplace fading channel with $b=1.5$

\section{Conclusions}

The BER of a 4-QAM/OFDM device in AWGN over a Laplace fading channel has been calculated, and the BER performance for this channel is investigated in this paper. Simulated results of the 4QAM/OFDM system in AWGN over Laplace fading channel were compared to the conventional 4QAM/OFDM system in AWGN over Rayleigh fading channel under various scenarios of the scale parameter of the Laplace distribution.

At $20 \mathrm{~dB}$ of SNR, The BER performance was as shown in table 1

Table 1. BER vales with different scenario of scale

\begin{tabular}{cc}
\multicolumn{2}{c}{ parameter } \\
\hline Scale parameter & BER \\
1.5 & $0.5^{*} 10^{-3}$ \\
0.7 & $0.2 * 10^{-2}$ \\
0.3 & $0.1 * 10^{-1}$ \\
\hline
\end{tabular}

The table above shows that the BER decreases with an increase in the scale parameter, and the best performance of our system was at 1.5 of the scale parameter values when the channel impact is low.

\section{Acknowledgements}

The author extends his thanks and gratitude to Mustansiryah University for supporting this research.

\section{Conflict of interest}

The authors confirm that the publication of this research causes no conflict of interest.

\section{References}

1. A. Farzamnia, N. W. Hlaing, M. Mariappan, and M. K. Haldar, "BER comparison of OFDM with M-QAM modulation scheme of AWGN and Rayleigh fading channels," 2018 9th IEEE Control and System Graduate Research Colloquium, ICSGRC 2018 - Proceeding, no. August, pp. 54-58, 2018, doi: 10.1109/ICSGRC.2018.8657503.

2. Richard van Nee and Ramjee Prasad, OFDM Wireless Multimedia Communications. Artech House universal personal communications library, 2000.

3. A. G. Armada, "Understanding the effects of phase noise in Orthogonal Frequency Division Multiplexing (OFDM)," IEEE Transactions on Broadcasting, vol. 47, no. 2, pp. 153-159, 2001, doi: 10.1109/11.948268.

4. L. J. Cimini, "Analysis and Simulation of a Digital Mobile Channel Using Orthogonal Frequency Division Multiplexing," IEEE Transactions on Communications, vol. 33, no. 7, pp. 665-675, 1985, doi: 10.1109/TCOM.1985.1096357.

5. G. A. Al-Rubaye, C. C. Tsimenidis, and M. Johnston, "Non-binary LDPC coded OFDM in impulsive power line channels," in 2015 23rd European Signal Processing Conference (EUSIPCO), 2015, pp. 14311435.

6. G. A. Al-Rubaye, C. C. Tsimenidis, and M. Johnston, "Improved performance of TCOFDM-PLNC for PLCs using exact derived impulsive noise PDFs," IEEE International Conference on Communications Workshops, ICC Workshops 2017, no. Scpa, pp. 1271-1276, 2017, doi: 10.1109/ICCW.2017.7962833.

7. G. A. Al-Rubaye, C. C. Tsimenidis, and M. Johnston, "Low-density parity check coded orthogonal frequency division multiplexing for PLC in non-Gaussian noise using LLRs derived from effective noise probability 
density functions," IET Communications, vol. 11, no. 16, pp. 2425-2432, 2017, doi: 10.1049/iet-com.2017.0265.

8. G. A. Al-Rubaye, C. C. Tsimenidis, and M. Johnston, "Performance evaluation of $\mathrm{T}$ COFDM under combined noise in PLC with log-normal channel gain using exact derived noise distributions," IET Communications, vol. 13, no. 6, pp. 766-775, 2015, doi: 10.1049/iet-com.2018.6185.

9. G. A. Al-Rubaye, "Performance analysis of $M$-ary OQAM/FBMC with impact of nonlinear distortion over compound Rician $\mathrm{K}$-factor unshadowed $/ \kappa-\mu$ shadowed fad ing channels," IET Communications, vol. 15, no. 1, pp. 60-77, 2020, doi: $10.1049 / \mathrm{cmu} 2.12059$.

10. A. Lect and G. Abd, "Three Dimensional Concatenated Codes in OFDM Transmission System under a Rayleigh Fading Channel with Carrier Interference," 2008, vol. 12, no. 2, pp. 137-156.

11. A. L. G. A. A.-K. Al-Rubyai, "Diversity Combining for DS CDMA System in a Rayleigh Fading Channel with Three Spreading Codes," Journal of Engineering and Development, Vol. 11, No. 1, March, pp. 1-16, 2007.

12. M. K. Simon and M.-S. Alouini, Digital communication over fading channels, vol. 95. John Wiley \& Sons, 2005.

13. J. G. Proakis, Digital communications, Second edi. McGraw Hill Co. Ltd., 1989.

14. S. Kotz, T. J. Kozubowski, and K. Podgórski, The Laplace Distribution and Generalizations. Boston, MA: Birkhäuser Boston, 2001.

15. E. C. Hedberg, Statistical Distributions. John Wiley \& Sons, Inc., Hoboken, New Jersey, 2011.

16. John G. Proakis and Masoud Salehi, Digital Communications, 5th Editio. McGraw-Hill Companies, Inc., 2008.

17. K. D. Rao, Channel coding techniques for wireless communications,Second Edition. 2019.

18. O. Ur-Rehman and N. Zivic, Wireless communications. Cambridge University Press, 2018.
19. J. G. Proakis and Masoud Salehi, Communication Systems Engineering, 2nd Editio. Prentice-Hall, Inc. Upper Saddle River, New Jersey, 2001. 